\title{
EFICÁCIA DE UMA NOVA FORMULAÇÃO DE GLIFOSATO PARA O CONTROLE DE GRAMA-SEDA (Cynodon dactylon), EM POMAR DE CITROS.
}

\author{
GUSTAVO MARTINI ${ }^{2}$, AIMAR FRANCISCO FERRARI PEDRINHO JUNIOR ${ }^{3}$, GUILHERME VARGAS FELICI ${ }^{3}$, FAUSTO \\ MONTEIRO PIVA ${ }^{3}$, JULIO CEZAR DURIGAN ${ }^{4}$
}

RESUMO - Avaliou-se a eficácia do herbicida glifosato potássico, comparado ao sulfosato e ao glifosato amínico, utilizados como padrões comerciais. Os herbicidas foram aplicados em um pomar de laranja da variedade Pêra-rio, infestado por grama-seda (Cynodon dactylon) com aproximadamente 25 $\mathrm{cm}$ de altura, em 70\% da área. O experimento foi instalado no município de Taiúva-SP, em solo Podzólico Vermelho-Amarelo, distrófico, de textura arenosa, com relevo suave ondulado. Os tratamentos testados foram: glifosato potássico (ZAPP Qi) a 1,05; 2,10 e 2,50 kg e.a./ha, sulfosato (ZAPP) e glifosato amínico (ROUNDUP CS) a 1,98 e 2,16 kg e.a./ha, respectivamente, além da testemunha sem capina. Os herbicidas foram aplicados em pósemergência da planta daninha, com pulverizador costal, à pressão constante (mantida por $\mathrm{CO}_{2}$ comprimido) de $2,5 \mathrm{~kg} / \mathrm{cm}^{2}$, munido de barra com seis bicos de jato plano ("leque") DG 11002, com um consumo de calda equivalente a $200 \mathrm{~L} / \mathrm{ha}$. Os melhores resultados de controle foram obtidos com 2,5 $\mathrm{kg}$ e.a./ha do glifosato potássico, que também garantiu menor brotação da grama-seda. No entanto, a dosagem de 2,10 kg e.a./ha deste mesmo herbicida não foi menos eficaz que o sulfosato e o glifosato, com 1,98 e 2,16 kg e.a./ha, respectivamente.

Termos para indexação: glyphosate, grama-seda, citros

\section{EFFICACY OF A NEW FORMULATION OF GLYPHOSATE TO BERMUDAGRASS CONTROL (Cynodon dactylon) IN CITRUS ORCHARD.}

\begin{abstract}
The aim of this experiment was to evaluate the performance of the potash glyphosate formulation, compared with sulphosate and aminic glyphosate formulation, used with commercial patterns, applied in orange orchard of Pera-rio variety, on the bermudagrass The experiment was conducted in Taiúva - SP, on ultisol. The treatments were: potash glyphosate (ZAPP Qi) at 1.05, 2.10 and 2.50 Kg a.e./ha, sulphosate (ZAPP) and aminic glyphosate formulation (ROUNDUP CS) at 1.98 and $2.16 \mathrm{Kg}$ a.e./ha, respectively, along writh the control without weed. The herbicides were applied in postemergency with costal sprayer, constant pressure $\left(2,5 \mathrm{~kg} / \mathrm{cm}^{2}\right)$, with six nozzles DG 11002 . The equivalent spray volume was $200 \mathrm{~L} / \mathrm{ha}$. The best results were obtained with $2.50 \mathrm{~kg}$ a.e./ha rate of potash glyphosate guarantying smaller regrowth of the bermudagrass. However, the 2.10 $\mathrm{kg}$ a.e./ha rate guarantied as good control as sulphosate at 1.98 and glyphosate at $2.16 \mathrm{~kg}$ a.e./ha.
\end{abstract}

Index terms: glyphosate, bermudagrass, citrus

\section{INTRODUÇÃO}

A citricultura tem grande importância no contexto nacional, pois é geradora de riquezas aos setores industrial e agrícola, garantindo o crescimento e a manutenção de todos os segmentos que dela se cercam (Fernandes \& Ulian, 1999). Ela é uma das grandes fontes geradoras de divisas para o País com a exportação de suco concentrado congelado e tem relevante importância no contexto mundial por seu volume de produção e área plantada. O estado de São Paulo é o grande produtor de citros, respondendo por, aproximadamente, $80 \%$ da produção nacional (Ramos \& Durigan, 1996) e $25 \%$ da mundial (Silva, 2000).

O Brasil possui baixa produtividade, comparado aos demais países produtores (De Negri, 1988). Um dos fatores responsáveis por isto é a interferência das plantas daninhas nos pomares, que podem acarretar perdas de 10 a 50\% (Blanco \& Oliveira, 1978; Salgado et al., 2000). As plantas daninhas reduzem o crescimento e a produção das plantas cítricas, devido à competição pelos fatores essenciais limitados no ecossistema comum ou, então, pela ação alelopática de substâncias químicas excretadas pelas mesmas. Além disso, podem servir como hospedeiras intermediárias de pragas e patógenos (Haddad, 1993).

Atualmente, são empregados vários métodos de controle das plantas daninhas, evitando-se a competição e facilitando-se os tratos culturais (Silva, 2000). O uso de herbicidas destaca-se por ser o mais eficiente e rápido, disponibilizando a mão-de-obra na propriedade. Os herbicidas mais utilizados são os aplicados em pós-emergência, principalmente devido aos efeitos prejudiciais dos chamados herbicidas residuais em culturas perenes, como em citros (Haddad, 1993) e em eucalipto (Rodrigues et al., 1991), se aplicados erroneamente. Caetano (2000) afirma que o uso de herbicidas proporcionou redução na infestação das plantas daninhas em relação à gradagem e roçagem. Dentre os herbicidas aplicados em pós-emergência, os mais conhecidos entre os citricultores são: glifosato, sulfosato e paraquat (Silva, 2000).
Um dos grandes problemas nos pomares é a presença de plantas daninhas com propagação vegetativa, que são de difícil controle. Cynodon dactylon, que produz grande número de rizomas e estolões, é exemplo disto (Mitich, 1989). Devido a essas características, torna-se necessário o uso de herbicidas sistêmicos. Johnson (1995) demonstrou que as diferentes cultivares de Cynodon dactylon, como Commom, Cheyenne, Tropica e Sahara, têm sucetibilidades diferentes para um mesmo herbicida. Alguns herbicidas necessitam de aplicações sequienciais, feitas em plantas jovens e posteriormente em adultas, repetidas ao longo dos anos, para o bom controle de Cynodon dactylon (Grichar, 1995), sendo que o glyphosate é indicado para o seu controle.

Claus \& Behrens (1976) e Devine et al. (1983) trabalharam com o controle de Agropyron repens; Wyrill \& Burnside (1976) e Waldecker \& Wyse (1985), com o de Asclepias syriaca; Camacho \& Moshier (1991) e Mcwhorter \& Hanks (1993), com o de Sorghum halepense, e concluíram que o glifosato mata os rizomas e suas brotações devido à sua ação sistêmica.

Osipe (1995), comparando glifosato com sulfosato, na mesma dosagem (4,5 L/ha do p.c.), não observou diferenças significativas no controle de Cynodon dactylon. Para Cyperus rotundus L., também não se verificaram diferenças entre sulfosato e glifosato nas dosagens de 1,92 e $2,40 \mathrm{~kg} / \mathrm{ha}$ (Braz \& Braz, 1995).

Em Agropyron repens, as dosagens de 0,56; 0,84 e 1,12 kg/ha de glifosato proporcionaram controle completo dos rizomas (Claus \& Behrens, 1976). Em cana-de-açúcar, Richard Jr. (1997) constatou que o cultivo mecânico foi o método menos eficaz para o controle de Cynodon dactylon. Duas aplicações do herbicida glifosato (a $3,36 \mathrm{~kg} / \mathrm{ha}$ ), com intervalo de um mês, foi o tratamento que proporcionou melhores resultados.

Outros herbicidas possuem boa eficácia para o controle de Cynodon dactylon. Bedmar (1997) cita que haloxifop, a $180 \mathrm{~g} / \mathrm{ha}$, e quizalofop-p-etil, a $54 \mathrm{~g} / \mathrm{ha}$, proporcionaram os melhores controles de Cynodon dactylon em batata. Em soja, além destes, o cletodim (336 g/ 
ha) e o propaquizalofop ( $100 \mathrm{~g} / \mathrm{ha}$ ) apresentaram o mesmo nível de controle. Cletodim e fenoxaprop, aplicados seqüencialmente, em duas épocas, fluazifop-p e quizalofop com aplicações únicas em pós-emergência inicial e setoxidim, aplicado uma ou duas vezes, foram os tratamentos que apresentaram os melhores controles de Cynodon dactylon em amendoim (Grichar, 1995). No grupo das imidazolinonas, o herbicida imazaquim, aplicado a $0,42 \mathrm{~kg} / \mathrm{ha}$, controlou razoavelmente esta planta daninha, porém os melhores resultados foram obtidos com o imazapir a $0,06 \mathrm{~kg} / \mathrm{ha}$ (Goatley Jr. et al., 1993).

Oren et al. (1984), citados por Haddad (1993), mostraram que o herbicida glifosato, na

concentração de $1,5 \%$ do produto comercial, aplicado com bicos rotativos, em volumes variáveis de 50 a $130 \mathrm{~L} / \mathrm{ha}$ de calda, controlou satisfatoriamente Cynodon dactylon. Singh \& Tucker (1983) aumentaram o controle de Cynodon dactylon com o aumento na dosagem $(0,56$ para $2,24 \mathrm{~kg} / \mathrm{ha}$ ) de glifosato.

O glifosato proporciona ótimo controle para a maioria das plantas daninhas com propagação vegetativa, porém apresenta baixa eficácia para algumas, tais como Commelina virginica L. (Lorenzi, 1986; Durigan, 1988). Visando-se a aumentar a eficácia deste herbicida, realizase a sua mistura com outros herbicidas (Galli, 1991). Neste sentido, Ramos \& Durigan (1996) observaram que o melhor controle de Commelina virginica $\mathrm{L}$. se deu com a mistura pronta de glifosato $+2,4$ $\mathrm{D}$ amina, nas dosagens de $0,65+0,81 \mathrm{~kg} / \mathrm{ha}$. Timossi et al. (2000) obtiveram bom controle de trapoeraba aplicando glifosato com imazamox ou carfentrazone.

Atualmente, o glifosato é uma das melhores alternativas para o controle químico de Cynodon dactylon, pois tem boa eficácia no controle, o menor preço em relação aos demais, é pouco tóxico ao homem e ao ambiente, e é de aplicação fácil e segura, por ser em pós-emergência protegida e dirigida.

No presente experimento, objetivou-se a avaliação da eficácia da formulação em sal potássico do glifosato, em três dosagens, comparadas ao sulfosato e ao sal amínico do glifosato, que são considerados padrões para o controle de grama-seda em pós-emergência.

\section{MATERIAISEMÉTODOS}

O experimento foi instalado e conduzido no Sítio São Judas Tadeu, municípo de Taiúva, Estado de São Paulo, com latitude de $21^{\circ} 07^{\circ}$ $\mathrm{S}$, longitude $48^{\circ} 21^{\prime} \mathrm{W}$ e altitude de $600 \mathrm{~m}$. Iniciou-se em 13-01-2000 e foi concluído em 25-02-2000.

O solo é classificado como Podzólico Vermelho-Amarelo, distrófico, horizonte B textural, textura arenosa, com relevo ondulado (Centurion, 1998).

Na região onde se localizou a área experimental, a temperatura média do mês mais quente (janeiro ou dezembro) é de $24,3^{\circ} \mathrm{C}$, e do mais frio (junho), de $18,6^{\circ} \mathrm{C}$. Também são características do clima regional, a média anual de precipitação de $1388,0 \mathrm{~mm}$ e médias anuais de temperaturas máxima e mínima de $29,4^{\circ} \mathrm{C}$ e $17,0^{\circ} \mathrm{C}$, respectivamente.

O experimento foi instalado a campo, no delineamento experimental de blocos casualizados, com seis tratamentos, em quatro repetições, totalizando 24 parcelas experimentais, em um pomar de laranjas da variedade Pêra-Rio, espaçada de três metros entre plantas e cinco metros entre linhas, com 5 anos de idade. As dimensões da parcela foram $3 \times 3 \mathrm{~m}$.

Os herbicidas testaods, com suas respectivas formulações e dosagens, assim como a testemunha sem aplicação de herbicidas, estão relacionados na Tabela 1.

Os herbicidas foram aplicados em pós-emergência das plantas daninhas, com pulverizador costal, à pressão constante (mantida pelo $\mathrm{CO}_{2}$ comprimido) de $2,5 \mathrm{~kg} / \mathrm{cm}^{2}$, munido de barra com seis bicos de jato plano ("leque") DG 11002 e consumo de calda equivalente a $200 \mathrm{~L} / \mathrm{ha}$.

$\mathrm{Na}$ aplicação dos herbicidas, o solo encontrava-se com excelente umidade, a temperatura ambiente era de $28,5^{\circ} \mathrm{C}$, a temperatura do solo (a $5 \mathrm{~cm}$ de profundidade) de $29,5{ }^{\circ} \mathrm{C}$, a umidade relativa do ar de
$68 \%$, os ventos intermitentes e fracos, com nebulosidade aproximada de $5 \%$. A grama-seda (Cynodon dactylon) predominava na área experimental, com $70 \%$ de infestação e 20 a $25 \mathrm{~cm}$ de altura.

TABELA 1 - Formulações e dosagens dos herbicidas, assim como a testemunha sem aplicação de herbicidas. Taiúva - SP, 2000.

\begin{tabular}{|c|c|c|}
\hline \multirow{2}{*}{ Tratamentos } & \multicolumn{2}{|c|}{ Dosagens } \\
\hline & (kg e.a./ha) & (L p.c./ha) \\
\hline 1. glifosato potássio ${ }^{(1)}$ & 1,05 & 2,1 \\
\hline 2. glifosato potássio & 2,10 & 4,2 \\
\hline 3. Glifosato potássio & 2,50 & 5,0 \\
\hline 4. sulfosato ${ }^{(2)}$ & 1,98 & 6,0 \\
\hline 5. glifosato amínico ${ }^{(3)}$ & 2,16 & 6,0 \\
\hline 6. Testemunha sem herbicida & - & - \\
\hline
\end{tabular}

$\begin{array}{ll}{ }^{(1)} \text { ZAPP QI } & \text { e.a. } \text { - equivalente ácido } \\ { }^{(2)} \text { ZAPP } & \text { p.c. }- \text { produto comercial } \\ { }^{(3)} \text { ROUNDUPCS } & \end{array}$

Foram realizadas avaliações visuais, atribuindo-se porcentagens de controle em relação à testemunha, aos 3; 7; 14;21;28 e 43 dias após a aplicação (DAA). O controle dessa planta daninha foi atribuído visualmente em relação à testemunha sem aplicação de herbicidas do respectivo bloco, onde se encontrava a parcela avaliada. As notas de controle variaram de 0 a $100 \%$, sendo $0 \%$ considerado sem injúria visível e $100 \%$ a necrose total dos tecidos das plantas de grama-seda. Os dados de controle foram transformados em arc-sen v\%, para a análise estatística.

\section{RESULTADOSE DISCUSSÃO}

As médias das percentagens de controle da grama-seda, atribuídas visualmente, em várias épocas após a aplicação dos herbicidas, são apresentadas na Tabela 2.

O controle de grama-seda consolidou-se aos 28 dias após a aplicação, quando se atingiram porcentagens iguais ou superiores a $90 \%$ para a maioria dos tratamentos. Os resultados obtidos aos 43 dias mostram redução das porcentagens de controle em função das rebrotas que foram constatadas nesta época. Devido a essas rebrotas precoces, alguns autores, como Grichar (1995) e Richard Jr. (1997), ressaltaram a necessidade de realizar-se aplicações seqüenciais, visando-se ao melhor controle da planta e menor reinfestação na área.

O tratamento com glifosato potássico a $1,05 \mathrm{~kg}$ e.a./ha foi menos eficaz, em todas as avaliações, confirmando a necessidade de maiores dosagens para o controle desta planta daninha. A rebrota também foi mais evidente nesse tratamento. Goatley Jr. et al. (1993), trabalhando com o herbicida imazaquin na dosagem de $0,42 \mathrm{~kg}$ e.a./ha, não obtiveram bom controle da grama-seda, pois ele apenas inibiu o seu crescimento. Melhores resultados foram alcançados com a aplicação da mistura deste herbicida com o imazapir.

O glifosato potássico a $2,10 \mathrm{~kg}$ e.a./ha proporcionou controle estatisticamente igual aos padrões (sulfosato e glifosato), até 28 DAA, diferindo negativamente aos 43 DAA por causa da rebrota mais intensa nas plantas sujeitas a esse tratamento. A eficácia da maior dosagem $(2,50 \mathrm{~kg}$ e.a./ha) desta nova formulação do herbicida foi superior a sua mais baixa $(1,05 \mathrm{~kg}$ e.a./ha) durante todo o período de avaliação e, dos 21 aos 43 dias após a aplicação, em relação à dosagem imediatamente menor (2,10 kg e.a./ha). Dependendo da espécie a ser controlada, a dosagem de glifosato pode ser reduzida, como no caso de Agropyron repens, para a qual, dosagens entre 0,56 e $1,12 \mathrm{~kg}$ e.a./ha ocasionaram a morte total dos rizomas (Claus \& Behrens, 1976).

O sulfosato e o glifosato, utilizados como padrões no experimento, não apresentaram diferenças significativas entre si e com as do- 
sagens de 2,10 e 2,50 kg e.a./ha de glifosato potássico, para o controle da grama-seda. Singh \& Tucker (1983) também obtiveram aumento no controle desta planta daninha com o aumento na dosagem de 0,56 para $2,24 \mathrm{~kg}$ e.a./ha de glifosato.

Aos 43 dias após a aplicação, apenas o tratamento com glyphosate potássico a 2,50 kg e.a./ha manteve controle superior a $90 \%$, porém sem diferir estatisticamente nos padrões.

Pelos dados obtidos, é possível indicar o uso do glifosato potássico a $2,10 \mathrm{~kg}$ e.a./ha para o controle de grama-seda, sem perda de eficácia em relação ao sulfosato a $1,98 \mathrm{~kg}$ e.a./ha e ao glifosato amínico a $2,16 \mathrm{~kg}$ e.a./ha. Isto levaria a uma redução na dosagem do produto comercial de 6,0 para 4,2 L/ha. No entanto, este tratamento permite a rebrota, e a reinfestação é mais precoce, com necessidade de reaplicação para a manutenção dos bons níveis de controle.

A grande quantidade de estolões e rizomas por onde o herbicida tem de distribuir-se após ser absorvido pelas folhas, é uma das razões para a necessidade de maiores dosagens para a obtenção de resultados consistentes de controle. Devido a isso, a dosagem maior de glyphosate potássico (2,50 kg e.a./ha) proporcionou melhor controle $(97,2 \%)$ aos 28 DAA e menor rebrota aos 43 DAA. Considerando-se que ela representa economia de 1,0 L/ha do produto comercial em relação aos padrões, novos estudos seriam necessários para avaliar-se os benefícios da equivalência de dosagens comerciais, apesar da maior quantidade de ativo dispendida neste tratamento.

No entanto, além da dose do herbicida, outros fatores podem interferir no controle de plantas daninhas com propagação vegetativa, como a temperatura (Devine et al., 1983), tamanho das gotas e volume de aplicação (McWhorter \& Hanks, 1993).

Com base nos resultados obtidos, torna-se fundamental um estudo da relação custo/benefício envolvendo dosagens, níveis e períodos de controle, além da quantidade de rebrotas e rapidez de reinfestação da área.

TABELA 2 - Médias das porcentagens de controle da grama-seda (Cynodon dactylon L.) em citros, atribuídas visualmente, em várias épocas após a aplicação dos herbicidas, em pós-emergência. Taiúva-SP, 2000.

\begin{tabular}{|c|c|c|c|c|c|c|}
\hline \multirow{2}{*}{ Tratamentos } & \multirow{2}{*}{$\begin{array}{l}\text { Dosagens } \\
\text { e.a. (kg/ha) }\end{array}$} & \multicolumn{5}{|c|}{ DIAS APÓS APLICAÇÃO } \\
\hline & & 7 & 14 & 21 & 28 & 43 \\
\hline 1. glifosasto potássio ${ }^{(I)}$ & 1,05 & $28,2^{a} \mathrm{~B}(22,5)^{b}$ & $47,2 \mathrm{~B}(53,7)$ & $57,7 \mathrm{C}((71,2)$ & $61,1 \mathrm{C}(76,2)$ & $54,1 \mathrm{~B}(65,0)$ \\
\hline 2. glifosato potássio & 2,10 & $32,4 \mathrm{AB}(28,7)$ & $58,5 \mathrm{AB}(72,5)$ & $68,4 \mathrm{~B}(86,2)$ & $71,9 \mathrm{~B}(90,0)$ & $64,5 \mathrm{~B}(80,0)$ \\
\hline 3. glifosato potássio & 2,50 & $46,1 \mathrm{~A}(51,2)$ & $69,3 \mathrm{~A}(83,7)$ & $78,9 \mathrm{~A}(95,0)$ & $82,1 \mathrm{~A}(97,2)$ & $75,5 \mathrm{~A}(91,2)$ \\
\hline 4. sulfosato(t) & 1,98 & $42,0 \mathrm{AB}(45,0)$ & $65,3 \mathrm{~A}(82,5)$ & $74,3 \mathrm{AB}(92,5)$ & $74,3 \mathrm{AB}(92,5)$ & $72,0 \mathrm{~A}(88,7)$ \\
\hline 5. glifosato aminico ${ }^{(3)}$ & 2,16 & $45,0 \mathrm{~A}(50,0)$ & $65,5 \mathrm{~A}(82,5)$ & $75,7 \mathrm{AB}(93,7)$ & $76,9 \mathrm{AB}(94,5)$ & $69,4 \mathrm{~A}(86,2)$ \\
\hline 6. Testemunha sem herbicida & - & $0,0 C(45,0)^{c}$ & $0,0 \mathrm{C}(55,0)$ & $0,0 \mathrm{D}(58,7)$ & $0,0 \mathrm{D}(55,0)$ & $0,0 \mathrm{C}(46,2)$ \\
\hline F & & $23,24 *$ & $56,32^{* *}$ & $175,59^{* *}$ & $290,61^{* *}$ & $74,28 * *$ \\
\hline CV\% & & 22,3 & 13,7 & 7,6 & 5,9 & 11,8 \\
\hline $\operatorname{dms}(5 \%)$ & & 16,57 & 16,04 & 10,39 & 8,29 & 15,15 \\
\hline
\end{tabular}

e.a. - equivalente ácido; ${ }^{\mathrm{a}}$ Dados transformados em arc-sen $\sqrt{ } \% ;{ }^{\mathrm{b}}$ Dados originais; ' Porcentagem de infestação; (1) ZAPP Qi; (2) ZAPP; (3) ROUNDUPCS

\section{CONCLUSÕES}

Para as condições edafoclimáticas e de infestação da área experimental e com base nos resultados obtidos, pode-se concluir que a dosagem de $2,10 \mathrm{~kg}$ e.a./ha de glifosato potássico pode ser indicada para o controle de grama-seda, sem perda de eficácia em relação ao sulfosato e glifosato, com 1,98 e 2,16 kg e.a./ha, respectivamente. A maior dosagem (2,5 kg e.a./ha) desta formulação foi a que garantiu menor brotação da planta daninha.

\section{REFERÊNCIASBIBLIOGRÁFICAS}

BEDMAR, F. Bermudagrass (Cynodon dactylon) control in sunflower (Helianthus annuus) soybean (Glycine max) and potato (Solanun tuberosum) with postemergence graminicides. Weed Technology, Champaign, v.11.p.683-688, 1997.

BLANCO, H. G.; OLIVEIRA, D. A. Estudos dos efeitos da época de controle do mato sobre a produção de citros e a composição da flora daninha. Arquivo Instituto Biológico de São Paulo, São Paulo, v. 45, n.1, p.25-26, 1978.

BRAZ, L.T.; BRAZ, B.A. Biological efficiency of sulfosate, compared to glyphosate for the control of seedge (Cyperus rotundus L.). Zeneca Agrícola, p.41-42, 1995

CAETANO, R.S.X. Dinâmica do banco de sementes e de populações de plantas daninhas na cultura dos citros (Citrus sinensis (L.) Osbeck.) submetida a diferentes sistemas de manejo. 2000. f.105. Tese (Doutorado em Fitotecnia) - Escola Superior de Agricultura "Luiz de Queiroz”, Universidade de São Paulo, Piracicaba, 2000.

CAMACHO, R.F.; MOSHIER, L.J. Absorption, translocation and activity of CGA-136872, DPX-V9360 and glyphosate in rhizome johnsongrass (Sorghum halepense). Weed Science, Champaign, v.39, p.354-357, 1991.

CLAUS, J.S.; BEHRENS, R. Glyphosate translocation and quackgrass rhizome bud kill. Weed Science, Champaign, v.24, p.149-152, 1976.

CENTURION, J.F. Caracterização e classificação dos solos da Faculdade de Ciências Agrárias e Veterinárias de Jaboticabal, 1998. 84f. Tese (Livre Docência) - Faculdade de Ciências Agrárias e Veterinárias, Universidade Estadual Paulista, Jaboticabal, 1998.

DE NEGRI, J.D. Práticas culturais para o aumento da produtividade dos citros. In: SIMPÓSIO DE CITRICULTURA, 3, 1988, Jaboticabal. Anais... p.205-219.

DEVINE, M.D.; BANDEEN, J.D.; MCKERSIE, B.D. Temperature effects on glyphosate absorption, translocation and distribution in quackgrass (Agropyron repens). Weed Science, Champaign, v.31. p.461-464, 1983.

DURIGAN, J.C. Controle químico de plantas daninhas na citricultura. Jaboticabal: FUNEP, 1988. p.18. (Boletim Informativo).

FERNADEZ, E.B.; ULIAN, L.F. Novos rumos da citricultura paulista. In: SECITAP. SEMANA DA CIÊNCIA E DA TECNOLOGIA AGROPECUÁRIA, 24., 1999, Jaboticabal. Resumos...p.26

GALLI, A.J.B. Avaliação da eficiência de glyphosate em mistura com diversos produtos, no controle de Commelina virginica (trapoeraba) em citros. In: CONGRESSOBRASILEIRODEHERBICIDAS EPLANTAS DANINHAS, 18., 1991, Brasília. Resumos... Brasília: SBHED, 1991.p.104-105.

GOATLEY Jr., J.M.; MADDOX, V.L.; WATKINS, R.M. Growth regulation of common bermudagrass (Cynodon dactylon) with Imazaquin and AC 263,222. Weed Technology, Champaign, v. 7, p.746-750, 1993.

GRICHAR, W.J. Comparison of postemergence herbicides for common bermudagrass (Cynodon dactylon) control in peanut (Arachis hypogea). Weed Technology, Champaign, v. 9, p.825-828, 1995.

HADDAD, A.C. Racionalização de herbicidas aplicados ao solo e em pós-emergência das plantas daninhas em pomares cítricos (Citrus sinensis (L.) Osbeck.). 1993. f.4-23. Monografia (Trabalho de Graduação em Agronomia) - Faculdade de Ciências Agrárias e Veterinárias, Universidade Estadual Paulista, Jaboticabal, 1993.

JOHNSON, B.J. Tolerance of four seeded commom bermudagrass (Cynodon dactylon) types to herbicides. Weed Technology, Champaign, v.9, p.794-800, 1995.

LORENZI, H. Manual de identificação e controle de plantas daninhas: plantio direto e convencional. 2. Editora Nova Odessa, 1986. 240p.

MCWHORTER, C.G.; HANKS, J.E. Effect of spray volume and pressure on postemergence johnsongrass (Sorghum halepense) control. Weed Technology, Champaign, v.7, p.304-310, 1993.

MITICH, L.W. Bermudagrass. Weed Technology, Champaign, v.3., p.433435, 1989. 
OREN, Y.; FELER, Z.; RUBIN, D. Weeds control with various glyphosate dosages and reduced spray volumes in citrus orchards. Phytoparasitica, Rehovot, v.10, n.4, p.273, 1982. In: Weed Abstracts, v.33, n.4, p.110, 1984. (Abstract 1050).

OSIPE, R. Control of Cynodon dactylon through the application of herbicides in postemergence, in different spray volumes. Zeneca Agrícola, v.2, p.45, 1995.

RAMOS, H.H.; DURIGAN, J.C. Avaliação da eficiência de uma mistura pronta de glyphosate mais 2,4-D no controle de Commelina virginica L. em citros. Planta Daninha, Campinas, v.14, n.1, p.33-41, 1996.

RICHARD Jr., E.P. Effects of fallow bermudagrass (Cynodon dactylon) control-programs on newly planted sugarcane (Saccharum spp. Hybrids). Weed Technology, Champaign, v. 11, p.677-682, 1997.

RODRIGUES, J.J.V. et al Efeitos de períodos de controle de convivência do capim-colonião (Panicum maximum Jacq.) na cultura do Eucalyptus. In: SEMINÁRIO TÉCNICO SOBRE PLANTAS DANINHAS E O USO DE HERBICIDAS EM REFLORESTAMENTO, 3., 1991, Belo Horizonte. Anais...p.43-54.

SALGADO, T.P.; ALVES, P.L.C.A.; KUVA, M.A.; MATTOS, E.D.; BRENDOLAN, R.A. Avaliação da eficiência do herbicida MON 78128 no controle de uma comunidade de plantas daninhas infestando a cultura da laranja. In: CONGRESSO BRASILEIRODA CIÊNCIA DAS
PLANTAS DANINHAS, 22., 2000, Foz do Iguaçu. Resumos.... p.412. SILVA, G. P. Eficiência do glyphosate e do sulfosate no controle de plantas daninhas na cultura dos citros Citrus sinensis (L.) Osbeck cv. Valência. 2000 - f 1-4 Monografia (Trabalho de Graduação em Agronomia) - Faculdade de Ciências Agrárias e Veterinárias,Universidade Estadual Paulista, Jaboticabal, 2000.

SINGH, M.; TUCKER, D.P.H. Effect of reduced glyphosate rates, spray volumes and delivery systems on Bermudagrass control in Flórida citrus groves. Procedings of the Florida State Horticultural Society, Winter Haven, v.96, p.34-37, 1983.

TIMOSSI, P.C.; ALVES, P.L.C.A.; MATTOS, E.D. Avaliação da eficácia de glyphosate, aplicado isolado ou em mistura com outros herbicidas, no controle de uma população de trapoeraba em pomares cítricos In: CONGRESSO BRASILEIRO DA CIÊNCIA DAS PLANTAS DANINHAS, 22., 2000, Foz do Iguaçu. Resumos... p. 437.

WALDECKER, M.A.; WYSE, D.L. Soil moisture effects on glyphosate absorption and translocation in common milkweed (Asclepias syriaca). Weed Science, Champaign, v.33, p.299-305, 1985.

WYRILL, J.B.; BURNSIDE, O.C. Absorption, translocation and metabolism of 2,4-D and glyphosate in common milkweed and hemp dogbane. Weed Science, Champaign, v.24, p.557-566, 1976. 